\title{
CAUCHY-LERAY-FANTAPPIÈ INTEGRAL IN LINEARLY CONVEX DOMAINS
}

\author{
A. S. Rotkevich*
}

UDC 517.55

\begin{abstract}
An important tool in analysis of functions of one complex variable is the Cauchy formula. However, in the case of several complex variables there is no unique and convenient formula of this sort. One can use the Szegö projection $S$, but the kernel of the operator $S$ has usually no closed form expression. Another choice is the Cauchy-LerayFantappié formula that has a rather closed form kernel for large classes of domains. In this paper, we prove the boundedness of the Cauchy-Leray-Fantappie integral for linearly convex domains as an operator on $L^{p}$ and $B M O$. Bibliography: 17 titles.
\end{abstract}

\section{INTRODUCTION}

This paper is devoted to study of regularity of the Cauchy-Leray-Fantappié integral in strictly linearly convex domains. The main ideas go back to the Hansson's paper [7] where a similar problem was considered for complex ellipsoids.

In analysis of holomorphic functions of one complex variable, the Cauchy formula is one of the main tools and objects of study. In the multi-dimensional case, we do not have such a universal and convenient formula to restore a function from its boundary values. It is possible to apply the Szegö projection $S$ which is defined as the orthogonal projection of the space $L^{2}(\partial \Omega)$ to the closed subspace $H^{2}(\Omega)$ generated by boundary values of holomorphic functions. The shortage of this approach is that, with the exception of a small number of special domains, one cannot represent the operator $S$ in a closed form.

Often, it is more convenient to consider operators that are generated by the Cauchy-Leray-Fantappié formula. The advantage of such operators is a relatively closed form for the kernel which one often can write using the function that determines the domain. To be exact, let us formulate the corresponding theorem.

Theorem 1. Let $\Omega$ be a bounded domain with a $C^{2}$-smooth boundary, and let a $C^{1}$-mapping $\eta: \partial \Omega \times \Omega \rightarrow \mathbb{C}^{n}$ satisfy the condition $\langle\eta(\xi, z), \xi-z\rangle \neq 0, \xi \in \partial \Omega, z \in \Omega$. Then the operator $K$ given by the formula

$$
K f(z)=\int_{\partial \Omega} \frac{f(\xi) \eta \wedge\left(\bar{\partial}_{\xi} \eta\right)^{n-1}}{\langle\eta, \xi-z\rangle^{n}}, \quad z \in \Omega,
$$

restores holomorphic functions from their boundary values .

Here (and in what follows, if we need this) we identify a vector $\eta \in \mathbb{C}^{n}$ and the differential $(1,0)$-form $\tilde{\eta}=\eta_{1} \partial \xi_{1}+\ldots+\eta_{n} \partial \xi_{n} ;$ in addition, $\langle w, z\rangle=w_{1} z_{1}+\ldots+w_{n} z_{n}$.

In connection with this statement, there arises the problem on the choice of a proper realization of formula (1) for a maximally wide class of domain. In the case of a strictly linearly convex domain with defining function $\rho$, one may take $\eta(\xi, z)=\partial \rho(\xi)$.

Our goal is to study this realization of the operator $K$; more exactly, we study its boundedness in the spaces $L^{p}$ and BMO. For strictly pseudoconvex domains (naturally, for a different realization of the kernel), the boundedness of the operator $K$ is proved in the paper [8]. Estimates on the Szegö kernel and the boundedness of the operator $S$ were studied for convex domains of finite type in [14], for pseudoconvex domains of finite type in $\mathbb{C}^{2}$ in [15], and for pseudoconvex domains with a single degenerate eigenvalue in [12]. All of these results are related to spaces that are constructed applying the usual Euclidean surface measure on $\partial \Omega$; in our case, it is more convenient to use the measure $d S$ generated by the form $d S=\partial \rho(\xi) \wedge(\bar{\partial} \partial \rho(\xi))^{n-1}$. We study this measure here for convenience since in this case, the measure $d \sigma$ is equivalent to the surface measure $d S$. As far as the author knows, Hansson [7] was the first to suggest using spaces constructed by the measure $d S$ in the study of boundedness of the operators $K$.

Define the function

$$
d(\xi, z)=|\langle\partial \rho(\xi), \xi-z\rangle|+|\langle\partial \rho(z), z-\xi\rangle|
$$

on $\partial \Omega \times \partial \Omega$.

${ }^{*}$ St.Petersburg State University, St.Petersburg, Russia, e-mail: rotkevichas@gmail.com.

Translated from Zapiski Nauchnykh Seminarov POMI, Vol. 401, 2012, pp. 172-188. Original article submitted June 14, 2012 . 
Section 2 is devoted to description of the geometry of the boundary for a strictly linearly convex domain. In particular, we show that $d$ is a quasi-metric and the measure $d S$ satisfies the condition of doubling with respect to it; more precisely, the volume of a ball of radius $r$ with respect to the quasi-metric $d$ is comparable to $r^{n}$. In addition, we show that the operator $K$ may be treated as a singular integral operator with a standard kernel.

We define the spaces $L^{p}$ and $H^{p}$ with respect to the measure $d S$. A function $f$ that is analytic in a domain $\Omega$ belongs to the space $H^{p}(\Omega)$ if and only if

$$
\|f\|_{H^{p}(\Omega)}^{p}=\sup _{r \rightarrow 0-} \int_{\partial \Omega_{-r}} f(\xi) d S_{-r}(\xi)<\infty,
$$

where $\Omega_{r}=\left\{z \in \mathbb{C}^{n}: \rho(z)<r\right\}$ and the measure $d S_{-r}$ is generated by the form $d S$.

Our basic result is as follows.

Theorem 2. The operator $K$ continuously maps the space $L^{2}(\partial \Omega)$ onto the space $H^{2}(\Omega)$.

According to the standard theory of Calderòn-Zygmund operators, this result expands to the spaces $L^{p}, p>1$.

Corollary 1. The operator $K$ continuously maps the space $L^{p}(\partial \Omega)$ onto the space $L^{p}, p>1$.

One can extend the function $K f$ to the boundary by the limits along normal directions; in this case, the operator $K$ is bounded as an operator onto $L^{2}(\partial \Omega)$, and we can consider the conjugate operator. In addition, defining the space BMO by the quasi-metric $d$ and the measure $d S$ on $\partial \Omega$, we establish the boundedness of the operator $K$ on this space.

Theorem 3. The operators $K$ and $K^{*}$ are bounded as mappings from the space $\mathrm{BMO}(\partial \Omega)$ to the space $\mathrm{BMO}(\partial \Omega)$.

Remark 1 (on notation). In what follows, we write $f \asymp g$ if there exists a constant $c>0$ independent of arguments of the values $f$ and $g$ and such that $c^{-1} f \leq g \leq c f$. Similarly, we write $f \lesssim g$ if there exists a constant $c>0$ such that $f \leq c g$.

\section{The boundary of a linearly CONVEX Domain as a homogeneous space}

In this section, we describe the geometry of the boundary for a strictly linearly convex domain $\Omega=\left\{z \in \mathbb{C}^{n}\right.$ : $\rho(z)<0\}$. In particular, we show that strict linear convexity implies strict linear convexity in the geometric sense, and thus, the distance from a point $z$ belonging to the boundary of the domain to the complex tangent plane at a point $\xi$ defined by the formula $|\langle\partial \rho(\xi), \xi-z\rangle|$ is separated from zero and, in addition, is estimated by $|\xi-z|^{2}$ if $\xi, z \in \partial \Omega$.

In what follows, we consider a domain $\Omega=\left\{z \in \mathbb{C}^{n}: \rho(z)<0\right\}$ that is determined by a $C^{2}$-smooth function $\rho$ such that $d \rho(\xi) \neq 0$ for $\xi \in \partial \Omega$. We denote by $\nu(\xi)=\frac{\partial \rho}{|\partial \rho|}$ the complex normal; let $n(\xi)=\frac{\operatorname{grad} \rho}{|\operatorname{grad} \rho|}$. We denote by $d^{2} \rho(\xi)[w]$ the action of the second differential of the function $\rho$ at a point $\xi \in \mathbb{C}^{n}$ on a vector $w \in \mathbb{C}^{n}$.

Definition 1. We denote by $\partial \Omega$ the tangent plane of the surface $\partial \Omega$ at a point $\xi$ :

$$
T_{\xi}^{\mathbb{R}}=\left\{z \in \mathbb{C}^{n}:(z-\xi) \perp \nu(\xi)\right\} .
$$

If the surface $S$ is regular, then $T_{\xi}^{\mathbb{R}}$ is a real $(2 n-1)$-dimensional hyperplane in $\mathbb{C}^{n}$; in this case, it contains a unique $(n-1)$-dimensional complex hyperplane; we call this hyperplane the complex tangent hyperplane and denote it by $T_{\xi}$.

Remark 2. If the domain $\Omega$ is nondegenerate, then

$$
\begin{aligned}
T_{\xi} & =\left\{z \in \mathbb{C}^{n}: \alpha(z-\xi) \in T_{\xi}^{\mathbb{R}}-\xi, \alpha \in \mathbb{C}\right\} \\
& =\left\{z \in \mathbb{C}^{n}: z-\xi, i(z-\xi) \in T_{\xi}^{\mathbb{R}}-\xi\right\}=\left\{z \in \mathbb{C}^{n}:\langle\partial \rho(\xi), z-\xi\rangle=0\right\} .
\end{aligned}
$$

It follows that if a complex line $\Gamma=\{\zeta+\alpha W: \alpha \in \mathbb{C}\}$ is tangent to the surface $S$, i.e., $\Gamma \subset T_{\xi}^{\mathrm{R}}$, then this line belongs to the complex tangent hyperplane $T_{\xi}$.

Definition 2. A domain $\Omega=\left\{z \in \mathbb{C}^{n}: \rho(z)<0\right\}$, where $\rho(\cdot) \in C^{2}\left(\mathbb{C}^{n}\right)$, is strictly linearly convex if

$$
d^{2} \rho(\xi)[z-\xi] \geq c|z-\xi|^{2}, \quad \xi \in \partial \Omega, \quad z \in T_{\xi}
$$


The differential form $(2 \pi i)^{-n} \partial \rho(\xi) \wedge(\bar{\partial} \partial \rho(\xi))^{n-1}$ determines a positive measure $d S$ on the boundary of the domain $\Omega$ that is equivalent to the surface measure since

$$
\partial \rho(\xi) \wedge(\bar{\partial} \partial \rho(\xi))^{n-1}=(n-1) ! \sum_{m=1}^{n}(-1)^{m-1} \frac{L(\rho)}{\rho_{\bar{\xi}_{m}}^{\prime}} d \bar{\xi}_{[m]} \wedge d \xi
$$

where $d \bar{\xi}_{[m]}=d \bar{\xi}_{1} \wedge \cdots \wedge d \bar{\xi}_{m-1} \wedge d \bar{\xi}_{m+1} \wedge \cdots \wedge d \bar{\xi}_{n}, d \xi=\bigwedge_{k=1}^{n} d \xi_{k}$, and the function $L(\rho)$ is strictly positive on $\partial \Omega$ (see [1]).

In addition, we introduce a measure $d V$ determined by the form $(2 \pi i)^{-n}(\bar{\partial} \partial \rho)^{n}$. Note that $(2 \pi i)^{-n}(\bar{\partial} \partial \rho)^{n}=$ $d\left((2 \pi i)^{-n} \partial \rho(\xi) \wedge(\bar{\partial} \partial \rho(\xi))^{n-1}\right)$ and $d V \sim d \lambda$, where $d \lambda$ is Lebesgue measure. For brevity, we use the notation $|U|=\int_{U} d S, U \subset \partial \Omega$.

Set $d(\xi, z)=|v(\xi, z)|+|v(z, \xi)|$, where $v(\xi, z)=\langle\partial \rho(\xi), \xi-z\rangle$, and denote $B_{\varepsilon}(z)=\{\xi \in \partial \Omega: d(\xi, z)<\varepsilon\}$. This metric combined with the measure $d S$ generates on $\partial \Omega$ the structure of a homogeneous space.

Lemma 1. If a domain $\Omega$ is strictly linearly convex, then the following estimates are valid:

(1) $\operatorname{dist}(z, \partial \Omega) \asymp \rho(z) \asymp|z-\xi|^{2}, z \in T_{\xi}, \xi \in \partial \Omega$;

(2) $\operatorname{dist}\left(\zeta, T_{\xi}\right) \asymp\left|\pi_{\xi}(\zeta)\right|^{2} \asymp|\xi-\zeta|^{2}, \xi, \zeta \in \partial \Omega$, where $\pi_{\xi}(\zeta)$ is the projection of $\zeta$ to the complex tangent plane at the point $\xi$;

(3) $|v(\zeta, \xi)| \asymp|v(\xi, \zeta)|, \zeta, \xi \in \partial \Omega$;

(4) $d(\zeta, \xi) \lesssim d(\xi, w)+d(w, \zeta), \zeta, \xi, w \in \partial \Omega$.

Proof. (1) Apply the Taylor formula to $\rho(z)$ :

$$
\begin{aligned}
\rho(z) & =\rho(\xi)+2 \operatorname{Re}\langle\partial \rho(\xi), z-\xi\rangle+\frac{1}{2} d^{2} \rho(\xi)\left[\frac{z-\xi}{|z-\xi|}\right] \cdot|z-\xi|^{2}+o\left(|z-\xi|^{2}\right) \\
& =\frac{1}{2} d^{2} \rho(\xi)\left[\frac{z-\xi}{|z-\xi|}\right] \cdot|z-\xi|^{2}+o\left(|z-\xi|^{2}\right) \asymp|z-\xi|^{2} .
\end{aligned}
$$

(2) Let $\zeta_{0} \in T_{\xi}$ be the point closest to the point $\zeta$; then $\pi_{\xi}(\zeta)=\zeta_{0}-\xi$ and $\operatorname{dist}\left(\zeta, T_{\xi}\right)=\left|\zeta-\zeta_{0}\right|=\operatorname{dist}\left(\zeta_{0}, \partial \Omega\right)$, which implies that $\operatorname{dist}\left(\zeta, T_{\xi}\right) \asymp\left|\zeta_{0}-\xi\right|^{2}=\left|\pi_{\xi}(\zeta)\right|^{2}$. Further, $|\zeta-\xi|^{2}=\left|\pi_{\xi}(\zeta)\right|^{2}+\operatorname{dist}\left(\zeta, T_{\xi}\right) \asymp\left|\pi_{\xi}(\zeta)\right|^{2}$.

(3) It is easily seen that $|v(\xi, z)| \asymp \operatorname{dist}\left(z, T_{\xi}\right) \asymp|z-\xi|^{2}$, which implies the required estimate.

(4) It is easy to deduce this property from the comparability of the values $d(\xi, \zeta)$ and $|\xi-\zeta|^{2}$ :

$$
d(\xi, \zeta) \asymp|v(\xi, \zeta)| \asymp|\xi-\zeta|^{2} \leq|\xi-w|^{2}+|w-\zeta|^{2} \lesssim d(\xi, \zeta)+d(\zeta, w) .
$$

We combine the property of $d$ formulated in item (4) of Lemma 1 with the facts that $d$ is symmetric and $d(\xi, z)$ is nondegenerate for $z \neq \xi$ to conclude that $d$ is a quasimetric. The measure $d S$ satisfies the doubling condition; in fact, even more can be said: the volume of a ball in the quasimetric $d$ is comparable to the $n$th degree of its radius.

Corollary 2. Denote by $B_{\varepsilon}\left(z_{0}\right)$ the ball of radius $\varepsilon$ (in the quasimetric d) centered at $z_{0} \in \partial \Omega$; then $\sigma\left(B_{\varepsilon}\left(z_{0}\right)\right) \asymp$ $\left|B_{\varepsilon}\left(z_{0}\right)\right| \asymp \varepsilon^{n}$.

Proof. Note that in complex-tangent directions, the distance in the quasimetric $d$ is comparable to the square root of the Euclidean distance; more precisely, $\operatorname{dist}\left(z, T_{z_{0}}\right) \asymp\left|\pi_{z_{0}}(z)\right|^{2} \asymp\left|v\left(z_{0}, z\right)\right| \asymp d\left(z, z_{0}\right)$. Hence, applying the projection to the tangent plane as a local parametrization of the set $\partial \Omega$, we conclude that

$$
\sigma\left(B_{\varepsilon}\left(z_{0}\right)\right) \asymp\left|B_{\varepsilon}\left(z_{0}\right)\right| \asymp \varepsilon^{1 / 2(2 n-2)} \varepsilon=\varepsilon^{n} .
$$

Thus, the measure $d S$ with respect to the quasimetric $d$ satisfies the doubling condition, and the space $(\partial \Omega, d, d S)$ is homogeneous. In what follows, we need the following two lemmas.

Lemma 2. The following estimates hold uniformly in $z \in \partial \Omega$ :

(1) $\int_{\substack{|v(z, \xi)|<h, \xi \in \Omega}} d V(\xi) \lesssim h^{n+1}$

(2) $\int_{\substack{|v(\xi, z)|<h, \xi \in \Omega^{c}}} d V(\xi) \lesssim h^{n+1}$. 
Proof. Let us prove, for example, item (2):

$$
\int_{\substack{|v(\xi, z)|<h, \xi \in \Omega^{c}}} d V(\xi) \asymp \int_{0}^{\varepsilon} \int_{|v(\zeta, z)|<\varepsilon-t} d S(\zeta) d t \asymp \int_{0}^{\varepsilon}(\varepsilon-t)^{n} d t \asymp \varepsilon^{n+1} .
$$

Lemma 3. Let $\alpha, \beta>0$ and $\beta-\alpha>-1$. Then the following estimates hold uniformly in $z \in \partial \Omega$ :

(1) $\int_{|v(\xi, z)|>h} \frac{d S(\xi)}{d(\xi, z)^{n+\alpha}} \lesssim h^{-\alpha}$;

(2) $\int_{\Omega} \frac{(-\rho(\xi))^{\beta-\alpha}}{|v(z, \xi)|^{n+1-\alpha}} d V(\xi) \lesssim 1$.

Proof. Let us check item (1). For this purpose, we decompose the set $\{\xi \in \partial \Omega: d(\xi, z)>h\}$ into setsa $\left\{\xi \in \partial \Omega: 2^{j-1} h<d(\xi, z) \leq 2^{j} h\right\}, j=1, \ldots, \infty$. Then

$$
\int_{|v(\xi, z)|>h} \frac{d S(\xi)}{d(\xi, z)^{n+\alpha}} \leq \sum_{j=1}^{\infty}\left(2^{j} h\right)^{-(n+\alpha)} \int_{|v(\xi, z)|<2^{j} h} d S(\xi) \lesssim h^{-\alpha} \sum_{j=1}^{\infty} 2^{-\alpha j} \lesssim h^{-\alpha} .
$$

To prove item (2), take $h>0$ and decompose the domain $\Omega$ into stripes: $\Omega=\bigcup_{j=0}^{N(h)} W_{j}$, where $W_{j}=\{\xi \in \Omega$ : $\left.2^{j-1} h \leq|v(z, \xi)|<2^{j} h\right\}, j=1, \ldots, N(h), W_{0}=\{\xi \in \Omega:|v(\xi, z)|<h\}$ and $N(h) \lesssim \log \frac{1}{h}$. We have shown that the linear convexity implies the estimate $|\rho(\xi)| \leq \operatorname{dist}\left(\xi, T_{z}\right) \lesssim|v(z, \xi)|$; taking this estimate into account, we conclude that

$$
\begin{aligned}
\int_{\Omega \backslash W_{0}} \frac{(-\rho(\xi))^{\beta-\alpha}}{|v(z, \xi)|^{n+1-\alpha}} d V(\xi) & \lesssim \sum_{j=1}^{N(h)} \int_{W_{j}} \frac{(-\rho(\xi))^{\beta-\alpha}}{|v(z, \xi)|^{n+1-\alpha}} d V(\xi) \\
& \lesssim \sum_{j=1}^{N(h)}\left(2^{j} h\right)^{-n-1+\alpha} \int_{|v(z, \xi)|<2^{j} h}(-\rho(\xi))^{\beta-\alpha} d V(\xi) \\
& \lesssim \sum_{j=1}^{N(h)}\left(2^{j} h\right)^{-n-1+\alpha} \int_{0}^{2^{j} h} \int_{\substack{\xi \in \partial \Omega_{-t},|v(\xi, z)|<2^{j} h}} t^{\beta-\alpha} d S_{-t}(\xi) d t \\
& \lesssim \sum_{j=0}^{N(h)}\left(2^{j} h\right)^{-n-1+\alpha}\left(2^{j} h\right)^{(\beta-\alpha+1)} 2^{j}\left(2^{j} h\right)^{n} \lesssim h^{\beta} \sum_{j=0}^{N(h)} 2^{\beta j} \lesssim h^{\beta} \log \frac{1}{h} \lesssim 1 .
\end{aligned}
$$

Hence, $\int_{\Omega} \frac{(-\rho(\xi))^{\beta-\alpha}}{|v(z, \xi)|^{n+1-\alpha}} d V(\xi) \leq \sup _{h>0} \int_{\Omega \backslash W_{0}} \frac{(-\rho(\xi))^{\beta-\alpha}}{|v(z, \xi)|^{n+1-\alpha}} d V(\xi) \lesssim 1$.

\section{BOUNDEDNESS OF THE OPERATOR}

In the notation introduced in the previous section, the operator $K$ is defined as follows:

$$
K f(z)=\int_{\partial \Omega} \frac{f(\xi) d S(\xi)}{v(\xi, z)^{n}}, z \in \Omega .
$$

This operator restores holomorphic functions that are continuous up to the boundary from their boundary values. Indeed, $K f=f$ for any function $f \in H^{1}(\Omega)$ since the intersection $H^{1}(\Omega) \cap C^{1}(\bar{\Omega})$ is dense in $H^{1}(\Omega)$.

In this section, we show that the operator $K$ acts boundedly from $L^{2}(\partial \Omega)$ to $H^{2}(\Omega)$. We prove this statement using a modification of the $T 1$-theorem for homogeneous spaces described in [5]. After that, we show that both the operator $K$ and its adjoint act boundedly from BMO to BMO.

First we formulate the $T 1$-theorem in the form in which we use it (see [5] for details). For this purpose, we need several definitions concerning singular integral operators. Recall that the quasimetric $d$ and measure $d S$ 
generate on $\partial \Omega$ the structure of a homogeneous space in which the volume of the ball $B_{r}(z)$ is comparable with $r^{n}$; this motivates the following definitions.

Definition 3. We call a kernel $k: \partial \Omega \times \partial \Omega \backslash\{\xi=z\} \rightarrow \mathbb{C}$ standard if there exist constants $A$ and $\delta>0$ such that

$$
|k(\xi, z)| \leq \frac{A}{d(\xi, z)^{n}}
$$

and

$$
|k(\xi, z)-k(\xi, w)|+|k(\xi, w)-k(z, w)| \leq A \frac{d(\xi, w)^{\delta}}{d(\xi, z)^{n+\delta}}
$$

for all $\xi, z, w \in \partial \Omega$ such that $d(\xi, z) \geq A d(\xi, w)$.

Remark 3. If we take the constant $A>0$ large enough (for example, if $A=2 c_{d}$, where $c_{d}$ is the constant of the quasimetric), then the condition $d(\xi, z) \geq A d(\xi, w)$ implies the relation $d(\xi, z) \asymp d(w, z)$.

Definition 4. Let $k$ be a standard kernel. An operator $T$ that acts from the space $C^{\infty}(\partial \Omega)$ to the space of distributions on $\partial \Omega$ by the formula

$$
\langle T f, g\rangle=\int_{\partial \Omega} \int_{\partial \Omega} k(\xi, z) f(z) g(\xi) d S(z) d S(\xi),
$$

is called a singular integral operator with kernel $k$.

Definition 5. We define the formal adjoint operator $T^{t}$ of an operator $T$ as an operator acting by the following rule:

$$
\left(\varphi, T^{t} \psi\right)=(T \varphi, \psi)
$$

Define the class $A(\delta, w, \varepsilon)$ as the class of smooth functions $\varphi$ that are concentrated on the ball $B_{\varepsilon}(w)$ and satisfy the estimate

$$
|\varphi(\xi)-\varphi(z)| \leq \frac{d(\xi, z)^{\delta}}{\varepsilon^{\delta}} .
$$

Definition 6. We say that an operator $T$ is weakly bounded if there exists a $\delta>0$ such that the estimate

$$
(T \varphi, \psi) \lesssim\left|B_{\varepsilon}(w)\right|
$$

is valid for all $w \in \partial \Omega, \varepsilon>0$, and for any functions $\varphi, \psi \in A(\delta, w, \varepsilon)$.

The T1-theorem states the following.

Proposition 1. A singular operator $T$ defined on a homogeneous space is bounded in $L^{2}$ if and only if this operator is weakly bounded and $T 1, T^{t} 1 \in \mathrm{BMO}$.

Proof of Theorem 2. On the whole, this proof repeats the proof of a similar result for ellipsoids given by Hansson in [7]. The only essential difference is in checking the fact that the kernel of the operator considered is standard.

First, we have to show that the kernel

$$
k(\xi, z)=\frac{1}{v(\xi, z)^{n}}=\frac{1}{\langle\partial \rho(\xi), \xi-z\rangle^{n}}
$$

is standard. Indeed, $|k(\xi, z)| \leq \frac{C}{d(\xi, z)^{n}}$. Let us check estimate (4):

$$
\left|\frac{1}{v(\xi, z)^{n}}-\frac{1}{v(\xi, w)^{n}}\right|=\left|\frac{v(\xi, z)^{n}-v(\xi, w)^{n}}{v(\xi, z)^{n} v(\xi, w)^{n}}\right| \lesssim \frac{|v(\xi, z)-v(\xi, w)|}{|v(\xi, z)|^{n+1}} \lesssim \frac{d(w, z)^{1 / 2}}{d(\xi, z)^{n+1 / 2}}
$$

since

$$
\begin{aligned}
|v(\xi, z)-v(\xi, w)| & =|\langle\partial \rho(\xi), z-w\rangle| \\
& \leq|\langle\partial \rho(\xi)-\partial \rho(w), z-w\rangle|+|\langle\partial \rho(w), z-w\rangle| \\
& \lesssim|\xi-w \| z-w|+d(z, w) \\
& \lesssim d(\xi, w)^{1 / 2} d(z, w)^{1 / 2}+d(\xi, w) \\
& \lesssim d(\xi, w)^{1 / 2} d(z, w)^{1 / 2}
\end{aligned}
$$


Similarly,

$$
\left|\frac{1}{v(\xi, z)^{n}}-\frac{1}{v(\zeta, z)^{n}}\right| \lesssim \frac{\left|v(\xi, z)^{n}-v(\zeta, z)^{n}\right|}{|v(\xi, z)|^{n+1}} \lesssim \frac{d(\xi, \zeta)^{1 / 2}}{d(\xi, z)^{n+1 / 2}}
$$

since

$$
\begin{aligned}
|v(\xi, z)-v(\zeta, z)| & =|\langle\partial \rho(\xi), \xi-z\rangle-\langle\partial \rho(\zeta), \zeta-z\rangle| \\
& \leq|\langle\partial \rho(\xi), \xi-\zeta\rangle|+|\langle\partial \rho(\xi)-\partial \rho(\zeta), \zeta-z\rangle| \\
& \lesssim d(\xi, \zeta)+|\xi-\zeta \| \zeta-z| \\
& \lesssim d(\xi, \zeta)+d(\xi, \zeta)^{1 / 2} d(\zeta, z)^{1 / 2} \\
& \lesssim d(\xi, \zeta)^{1 / 2} d(\xi, z)^{1 / 2} .
\end{aligned}
$$

Define the operator $K$ as an operator on $\partial \Omega$. For a point $z_{0} \in \partial \Omega$ we set $K f\left(z_{0}\right)$ to be equal the limit along the complex tangent direction. Such a limit does not always exist; nevertheless, if $f \in C^{1}$, then

$$
K f(z)=\int_{\partial \Omega} \frac{f(\xi)-f\left(z_{0}\right)}{v(\xi, z)^{n}} d S(\xi)+f\left(z_{0}\right)
$$

and we can pass to the limit under the integral sign, i.e.,

$$
K f\left(z_{0}\right)=\int_{\partial \Omega} \frac{f(\xi)-f\left(z_{0}\right)}{v\left(\xi, z_{0}\right)^{n}} d S(\xi)+f\left(z_{0}\right) .
$$

Thus, the operator $K$ is a singular integral operator with kernel $k(\xi, z)$.

Now we can apply the $T 1$-theorem to show that the operator $K$ is bounded on the space $L^{2}$. The operator $K$ restores holomorphic functions from their boundary values; hence, $K 1=1 \in$ BMO. Further we show that $K^{t} 1 \in L^{\infty}$. By the Stokes formula,

$$
\int_{\partial \Omega_{-r}} \frac{\partial \rho(z) \wedge(\bar{\partial} \partial \rho(z))^{n-1}}{v(\xi, z)^{n}}=\int_{\Omega_{-r}} \frac{(\bar{\partial} \partial \rho(z))^{n}}{v(\xi, z)^{n}} .
$$

Note that the right-hand side converges uniformly in $\xi$ as $r \rightarrow 0$; hence,

$$
\int_{\partial \Omega_{-r}} K f d S=\int_{\partial \Omega} f(\xi)\left(\int_{\Omega_{-r}} \frac{d V(\xi)}{v(\xi, z)^{n}}\right) d S(\xi)
$$

by the Fubini theorem.

If $f \in C^{\infty}$, then the function $K f$ is continuous up to the boundary, and, passing to the limit, we conclude that

$$
\int_{\partial \Omega} f \overline{K^{t} 1} d S=\int_{\partial \Omega} K f d S=\int_{\partial \Omega} f(\xi)\left(\int_{\Omega} \frac{d V(\xi)}{v(\xi, z)^{n}}\right) d S(\xi) .
$$

Hence,

$$
K^{t} 1(z)=\int_{\Omega} \frac{d V(\xi)}{\overline{v(\xi, z)}^{n}}, z \in \partial \Omega .
$$

In particular, $K^{t} 1 \in L^{\infty} \subset$ BMO. It remains to check that the operator $K$ is weakly bounded. For this purpose, we need the following lemma.

Lemma 4. If $z \in \partial \Omega$ and $\varepsilon>0$, then

$$
\int_{d(\xi, z)>\varepsilon} \frac{d S(\xi)}{v(\xi, z)^{n}} \lesssim 1
$$


Proof. Since $d(\xi, z) \lesssim|v(\xi, z)|$, the inequality

$$
\int_{d(\xi, z)>\varepsilon} \frac{d S(\xi)}{v(\xi, z)^{n}} \lesssim\left|\int_{|v(\xi, z)|>\varepsilon} \frac{d S(\xi)}{v(\xi, z)^{n}}\right|+\int_{\varepsilon \gtrsim|v(\xi, z)|>\varepsilon} \frac{d S(\xi)}{|v(\xi, z)|^{n}}
$$

holds. Clearly, the second integral on the right is bounded. To estimate the first integral, we apply the Stokes theorem for the domain $\left\{\xi \in \Omega^{c}:|v(\xi, z)|>\varepsilon, \rho(\xi)<1\right\}$. Since $\frac{\partial \rho(\xi) \wedge(\bar{\partial} \partial \rho(\xi))^{n-1}}{v(\xi, z)^{n}}$ is a closed form for $\xi \neq z$ and

$$
\int_{\rho(\xi)=1} \frac{\partial \rho(\xi) \wedge(\bar{\partial} \partial \rho(\xi))^{n-1}}{v(\xi, z)^{n}}=1
$$

we conclude that

$$
\int_{|v(\xi, z)|>\varepsilon} \frac{\partial \rho(\xi) \wedge(\bar{\partial} \partial \rho(\xi))^{n-1}}{v(\xi, z)^{n}}=1-\int_{\substack{|v(\xi, z)|=\varepsilon, \xi \in \Omega^{c}}} \frac{\partial \rho(\xi) \wedge(\bar{\partial} \partial \rho(\xi))^{n-1}}{v(\xi, z)^{n}} .
$$

Integrating over $\xi$ such that $|v(\xi, z)|=\varepsilon$, we replace

$$
1 / v(\xi, z)=\overline{v(\xi, z)} / \varepsilon^{2} .
$$

Then the Stokes theorem applied to the domain

$$
\left\{\xi \in \Omega^{c}:|v(\xi, z)|<\varepsilon\right\}
$$

implies that

$$
\begin{aligned}
& \int_{\substack{|v(\xi, z)|=\varepsilon, \xi \in \Omega^{c}}} \frac{\partial \rho(\xi) \wedge(\bar{\partial} \partial \rho(\xi))^{n-1}}{v(\xi, z)^{n}}=\frac{1}{\varepsilon^{2 n}} \int_{\substack{v(\xi, z) \mid=\varepsilon, \xi \in \Omega^{c}}} \overline{v(\xi, z)}^{n} \partial \rho(\xi) \wedge(\bar{\partial} \partial \rho(\xi))^{n-1} \\
& =\frac{1}{\varepsilon^{2 n}} \int_{|v(\xi, z)|<\varepsilon} \overline{v(\xi, z)}^{n} \partial \rho(\xi) \wedge(\bar{\partial} \partial \rho(\xi))^{n-1} \\
& +\frac{1}{\varepsilon^{2 n}} \int_{\substack{|v(\xi, z)|<\varepsilon, \xi \in \Omega^{c}}} d_{\xi} \overline{v(\xi, z)}^{n} \wedge \partial \rho(\xi) \wedge(\bar{\partial} \partial \rho(\xi))^{n-1} \\
& +\frac{1}{\varepsilon^{2 n}} \int_{\substack{|v(\xi, z)|<\varepsilon, \xi \in \Omega^{c}}} \overline{v(\xi, z)}^{n}(\bar{\partial} \partial \rho(\xi))^{n} .
\end{aligned}
$$

Since $\left|d_{\xi} \overline{v(\xi, z)}^{n} \wedge \partial \rho(\xi) \wedge(\bar{\partial} \partial \rho(\xi))^{n-1}\right| \lesssim\left|v(\xi, z)^{n}\right|$, we get the estimate

$$
\left|\int_{|v(\xi, z)|>\varepsilon} \frac{\partial \rho(\xi) \wedge(\bar{\partial} \partial \rho(\xi))^{n-1}}{v(\xi, z)^{n}}\right| \leq 1+\frac{1}{\varepsilon^{n}} \int_{|v(\xi, z)|<\varepsilon} d S(\xi)+\frac{1}{\varepsilon^{n+1}} \int_{\substack{|v(\xi, z)|<\varepsilon, \xi \in \Omega^{c}}} d V(\xi)+\frac{1}{\varepsilon^{n}} \int_{\substack{|v(\xi, z)|<\varepsilon, \xi \in \Omega^{c}}} d V(\xi),
$$

and the integral is bounded by Lemma 3 .

Now we check that the operator $K$ is weakly bounded. Take a function $\varphi \in A\left(\frac{1}{2}, w, \varepsilon\right)$ and a large enough constant $C$; then

$$
K \varphi(z)=\int_{d(\xi, w) \leq C \varepsilon} \frac{\varphi(\xi)-\varphi(z)}{v(\xi, z)^{n}} d S(\xi)+\varphi(z)\left(1-\int_{d(\xi, w)>C \varepsilon} \frac{1}{v(\xi, z)^{n}} d S(\xi)\right) .
$$


By the preceding lemma, the second summand is uniformly bounded. We estimate the first summand as follows:

$$
\left|\int_{d(\xi, w) \leq C \varepsilon} \frac{\varphi(\xi)-\varphi(z)}{v(\xi, z)^{n}} d S(\xi)\right| \lesssim \varepsilon^{1 / 2} \int_{d(\xi, w) \leq C \varepsilon} \frac{1}{d(\xi, z)^{n-1 / 2}} d S(\xi) \lesssim \frac{\varepsilon^{1 / 2}}{\varepsilon^{1 / 2}}=1,
$$

i.e., $\|K \varphi(z)\|_{\infty} \lesssim 1$ for all $\varphi \in A\left(\frac{1}{2}, w, \varepsilon\right)$. Hence, the operator $K$ is weakly bounded:

$$
|\langle K \varphi, \psi\rangle| \lesssim \int_{\partial \Omega}|\psi| d S=\int_{d(\xi, w)<\varepsilon}|\psi| d S \lesssim\left|B_{\varepsilon}(w)\right|, \quad \varphi, \psi \in A(1 / 2, w, \varepsilon) .
$$

The $T 1$-theorem implies that the operator $K$ defined on the boundary as above is extended to a bounded operator on $L^{2}(\partial \Omega)$. It is easily seen that this operator acts boundedly from $L^{2}(\partial \Omega)$ onto $H^{2}(\Omega)$. Indeed, take a function $f \in L^{2}(\partial \Omega)$ and approximate this function by a sequence of functions $f_{n} \in C^{1}$ in the $L^{2}$ norm. Then

$$
\int_{\partial \Omega_{-r}}\left|K f_{n}\right|^{2} d S \leq \int_{\partial \Omega}\left|K f_{n}\right|^{2} d S \lesssim \int_{\partial \Omega}\left|f_{n}\right|^{2} d S .
$$

Passing to the limit as $n \rightarrow \infty$, we get the relation

$$
\int_{\Omega_{-r}}|K f|^{2} d S \lesssim \int_{\partial \Omega}|f|^{2} d S
$$

Hence, $\|K f\|_{H^{2}} \leq\|f\|_{L^{2}}$, and the theorem is proved.

Let us define the adjoint operator $K^{*}$. For a function $f \in C^{1}$, the action of the operator on this function defines a function that is continuous up to the boundary, and we can extend $K f$ to the boundary. Note that functions of the class $H^{2}$ have normal boundary values almost everywhere, and $\|F\|_{H^{2}(\Omega)} \asymp\|F\|_{L^{2}(\partial \Omega)}$; thus, Theorem 2 implies that functions $K f(z)$ can be extended to the boundary of the domain, and the operator obtained is bounded in $L^{2}$. In addition, this limit operator coincides with the operator introduced in the proof of the theorem (since these two operators coincide on smooth functions). As an operator on $L^{2}$, it has an adjoint operator which we denote by $K^{*}$. The reasoning applied in the proof of Theorem 2 shows that $K^{*}$ is also a singular integral operator with kernel $1 \overline{v(\xi, z)}^{n}$.

Definition 7. A function $b \in L^{1}(\partial \Omega)$ belongs to the class BMO if the expression

$$
\|b\|_{\mathrm{BMO}}=\sup _{\substack{z_{0} \in \partial \Omega \\ \varepsilon>0}} \frac{1}{\left|B_{\varepsilon}\left(z_{0}\right)\right|} \int_{B_{\varepsilon}\left(z_{0}\right)}\left|b(z)-b_{B_{\varepsilon}\left(z_{0}\right)}\right| d S(z)+\int_{\partial \Omega}|b(z)| d S(z)
$$

is finite, where $b_{B_{\varepsilon}\left(z_{0}\right)}=\frac{1}{\left|B_{\varepsilon}\left(z_{0}\right)\right|} \int_{B_{\varepsilon}\left(z_{0}\right)} b(z) d S(z)$ is the mean value of the function $b$ on the ball $B_{\varepsilon}\left(z_{0}\right)$.

Note that our definition of the norm in the class BMO differs from the classical one, in which the expression for the norm only contains the first summand; in addition, the space BMO is factored by the subspace of constant functions. Due to this fact and the finiteness of the measure $d S$, our reasoning somewhat differs from the reasoning usually applied in similar situations; in particular, $K^{t} 1 \neq$ const in our case. For functions of the class BMO, the following John-Nierenberg inequality holds:

$$
\frac{1}{\left|B_{\varepsilon}\left(z_{0}\right)\right|} \int_{B_{\varepsilon}\left(z_{0}\right)}\left|b(z)-b_{B_{\varepsilon}\left(z_{0}\right)}\right|^{q} d S(z) \leq\|b\|_{\mathrm{BMO}}^{q}, \quad q>1 .
$$

Proof of Theorem 3. Our proof repeats the reasoning of the Hansson's paper [7]. Take a function $b \in \mathrm{BMO}$, fix a point $z_{0} \in \partial \Omega$, and represent $b$ in the form $b=b_{1}+b_{2}+b_{3}$, where $b_{1}=b_{B_{\varepsilon}\left(z_{0}\right)}$ and $b_{2}=\left(b-b_{1}\right) \chi_{B_{C \varepsilon}\left(z_{0}\right)}$, where $\chi_{B_{C \varepsilon}\left(z_{0}\right)}$ is the indicator of the set $B_{C \varepsilon}\left(z_{0}\right)$ and $C$ is a large enough constant depending on the constant in the triangle inequality for the quasimetric $d$. Then $K b_{1}=b_{1}$; hence,

$$
\frac{1}{\left|B_{\varepsilon}\left(z_{0}\right)\right|} \int_{B_{\varepsilon}\left(z_{0}\right)}\left|K b_{1}(z)-K b_{1 B_{\varepsilon}\left(z_{0}\right)}\right| d S(z)=0 .
$$


To estimate the function $K b_{2}$, we take into account that the operator $K$ is bounded in $L^{2}$ and apply the Jensen's inequality:

$$
\begin{aligned}
& \left(\frac{1}{\left|B_{\varepsilon}\left(z_{0}\right)\right|} \int_{B_{\varepsilon}\left(z_{0}\right)}\left|K b_{2}-\left(K b_{2}\right)_{B_{\varepsilon}\left(z_{0}\right)}\right| d S\right)^{2} \lesssim\left(\frac{1}{\left|B_{\varepsilon}\left(z_{0}\right)\right|} \int_{B_{\varepsilon}\left(z_{0}\right)}\left|K b_{2}\right| d S\right)^{2} \\
& \quad \lesssim \frac{1}{\left|B_{\varepsilon}\left(z_{0}\right)\right|} \int_{\partial \Omega}\left|K b_{2}\right|^{2} d S \lesssim \frac{1}{\left|B_{\varepsilon}\left(z_{0}\right)\right|} \int_{\partial \Omega}\left|b_{2}\right|^{2} d S \\
& =\frac{1}{\left|B_{\varepsilon}\left(z_{0}\right)\right|} \int_{B_{C \varepsilon}\left(z_{0}\right)}\left|b(z)-b_{B_{\varepsilon}\left(z_{0}\right)}\right|^{2} d S(z) \lesssim\|b\|_{\mathrm{BMO}}^{2} .
\end{aligned}
$$

Finally, estimating $K b_{3}$, we obtain the relations

$$
\begin{aligned}
& \frac{1}{\left|B_{\varepsilon}\left(z_{0}\right)\right|} \int_{B_{\varepsilon}\left(z_{0}\right)}\left|K b_{3}-\left(K b_{3}\right)_{B_{\varepsilon}\left(z_{0}\right)}\right| d S \\
& \lesssim \frac{1}{\left|B_{\varepsilon}\left(z_{0}\right)\right|^{2}} \int_{B_{\varepsilon}\left(z_{0}\right)}\left(\int_{B_{\varepsilon}\left(z_{0}\right)}\left(\int_{\partial \Omega}\left|\frac{1}{v(\zeta, z)^{n}}-\frac{1}{v(\zeta, w)^{n}}\right|\left|b_{3}(\zeta)\right| d S(\zeta)\right) d S(w)\right) d S(z) \\
& \lesssim \frac{1}{\left|B_{\varepsilon}\left(z_{0}\right)\right|^{2}} \int_{B_{\varepsilon}\left(z_{0}\right)} \int_{B_{\varepsilon}\left(z_{0}\right)} \int_{\partial \Omega} \frac{d(z, w)^{1 / 2}}{d(\zeta, z)^{n+1 / 2}}\left|b_{3}(\zeta)\right| d S(\zeta) d S(z) d S(w) \\
& \lesssim \varepsilon^{1 / 2} \int_{B_{C \varepsilon}^{c}\left(z_{0}\right)} \frac{\left|b(\zeta)-b_{B_{C \varepsilon}\left(z_{0}\right)}\right|}{d\left(\zeta, z_{0}\right)^{n+1 / 2}} d S(\zeta) \lesssim\|b\|_{\text {BMO }}
\end{aligned}
$$

since $d(\zeta, z) \lesssim d(z, w)$ and $d(\zeta, z) \asymp d\left(\zeta, z_{0}\right)$ if the constant $C>0$ is large enough; it is easy to get the last inequality dividing the integration domain into dyadic balls $B_{2^{j} C \varepsilon}\left(z_{0}\right)$. Thus, we have estimated the first term in the expression for the BMO-norm of the function $K b$. Let us estimate the second term, $\|K b\|_{L^{1}}$. We represent

$$
K b=K b_{\partial \Omega}+K\left(b-b_{\partial \Omega}\right)=b_{\partial \Omega}+K\left(b-b_{\partial \Omega}\right),
$$

the first term is estimated by $\|b\|_{\mathrm{BMO}}$, and the second term is estimated by $K b_{2}$.

A similar reasoning shows that the operator $K^{*}$ is bounded in BMO. The only difference is in estimation of the summand $K^{*} b_{1}$ since the function $K^{*} 1$ is not constant. Nevertheless, applying expression (12) for $K^{*} 1$, estimate (9), and Lemma 3 , one can show that the function $K^{*} 1$ is Hölder with exponent $1 / 2$ :

$$
\begin{aligned}
\left|K^{*} 1(z)-K^{*} 1(w)\right| & \lesssim \int_{\Omega}\left|\frac{1}{v(\xi, z)^{n}}-\frac{1}{v(\xi, w)^{n}}\right| d V(\xi) \\
& \lesssim \int_{\Omega} \frac{d(w, z)^{1 / 2}}{d(\xi, z)^{n+1 / 2}} d V(\xi) \lesssim d(w, z)^{1 / 2} .
\end{aligned}
$$

In addition, the estimate $\left|b_{1}\right| \leq\left\|b_{1}\right\|_{\text {BMO }} \log \frac{1}{\varepsilon}$ is valid (see [6]). It follows that

$$
\begin{aligned}
& \frac{1}{\left|B_{\varepsilon}\left(z_{0}\right)\right|} \int_{B_{\varepsilon}\left(z_{0}\right)}\left|K^{*} b_{1}(z)-\left(K^{*} b_{1}\right)_{B_{\varepsilon}\left(z_{0}\right)}\right| d S(z) \\
& \quad \lesssim \frac{\left|b_{1}\right|}{\left|B_{\varepsilon}\left(z_{0}\right)\right|^{2}} \int_{B_{\varepsilon}\left(z_{0}\right)} \int_{B_{\varepsilon}\left(z_{0}\right)}\left|K^{*} 1(z)-K^{*} 1(\xi)\right| d S(\xi) d S(z) \\
& \lesssim\left|b_{1}\right| \varepsilon^{1 / 2} \leq \varepsilon^{1 / 2} \log \frac{1}{\varepsilon}\|b\|_{\text {BMO }} \lesssim\|b\|_{\text {BMO } .}
\end{aligned}
$$

The summands $K^{*} b_{2}$ and $K^{*} b_{3}$ are estimated using the same reasoning as in the case of the operator $K$; we take into account that the operator $K^{*}$ is a singular operator with standard kernel $1 / \overline{v(\xi, z)}^{n}$. Finally, we conclude that the operator $K^{*}$ is bounded in BMO. 
This research was supported by the Chebyshev Laboratory, St. Petersburg State University (grant of the Government of Russia 11.G34.31.0026).

Translated by S. Yu. Pilyugin.

\section{REFERENCES}

1. L. A. Aizenberg and A. P. Yuzhakov, Integral Representations and Residues in Complex Analysis [Russian], Moscow (1979).

2. A. Rotkevich, "The Aizenberg formula in nonconvex domains and some of its applications," Zap. Nauchn. Semin. POMI, 389, 206-231 (2011).

3. J. D. McNeal, "The Bergman projection as a singular integral operator," J. Geom. Anal., 4, 91-103 (1994).

4. K. Adachi, Several Complex Variables and Integral Formulas, World Scientific (2007).

5. G. David, J. L. Journe, and S. Semmes, "Opérateurs de Calderón-Zygmund, fonctions para-accrétives et interpolation," Rev. Mat. Iber., 1, 1-56 (1985).

6. C. Fefferman and E. M. Stein, " $H^{p}$ spaces of several variables," Acta Mathematica, 129, 137-193 (1972). DOI: $10.1007 / \mathrm{BF} 02392215$.

7. T. Hansson, "On Hardy spaces in complex ellipsoids," Annales de l'institut Fourier, 49, 1477-1501 (1999). DOI:10.5802/aif.1727.

8. N. Kerzman and E. M. Stein, "The Szego kernel in terms of Cauchy-Fantappie kernels," Duke Math. J., 45, 197-224 (1978).

9. A. Korànyi and S. Vàgi, "Singular integrals on homogeneous spaces and some problems of classical analysis," Annali della Scuola Normale Superiore di Pisa - Classe di Scienze, Sér. 3, 25, 575-648 (1971).

10. L. Lanzani and E. M. Stein, "Szegö and Bergman projections on non-smooth planar domains," J. Geom. Anal., 14, 63-86 (2004). DOI: 10.1007/BF02921866.

11. L. Lanzani and E. M. Stein, "The Bergman projection in $L^{p}$ for domains with minimal smoothness" (2012), arXiv: $1201.4148 \mathrm{v} 1$.

12. M. Machedon, "Szego kernels on pseudoconvex domains with one degenerate eigenvalue," Ann. Math., 2nd Ser., 128, 619-640 (1988).

13. J. D. McNeal and E. M. Stein, "Mapping properties of the Bergman projection on convex domains of finite type," Duke Math. J., 73, 177-199 (1994).

14. J. D. McNeal and E. M. Stein, "The Szegö projection on convex domains," Mathematische Zeitschrift, 224, 519-553 (1997). DOI: 10.1007/PL00004593.

15. A. Nagel, J. P. Rosay, E. M. Stein, and S. Wainger, "Estimates for the Bergman and Szego kernels in $C^{2}$," Ann. Math., 129, 113-149 (1989).

16. W. Rudin, Function Theory in the Unit Ball of $\mathbb{C}^{n}$, Grundlehren der mathematischen Wissenschaften, 241, Springer-Verlag (1980).

17. E. L. Stout, " $H^{p}$-functions on strictly pseudoconvex domains," Amer. J. Math., 98, 821-852 (1976). 